\section{A21 ISOTYPES OF ANTICYCLIC CITRULLINATED PEPTIDE AND IGM AND IGA RHEUMATOID FACTOR IN SUDANESE PATIENTS WITH RHEUMATOID ARTHRITIS}

Amir I Elshafie, ${ }^{1,2}$ Sahwa A Norein, ${ }^{3}$ Mohammed Mullazehi,, Belinda Lind Norin, Mouwahib Al-Edreese, ${ }^{4}$ Elnour M Elagib, ${ }^{5}$ Musa A M Nur, ${ }^{4}$ Johan Rönnelid ${ }^{1}{ }^{1}$ Unit of Clinical Immunology, Uppsala University, Uppsala, Sweden; ${ }^{2}$ Department of Clinical Pathology, Alribat University Hospital, Khartoum, Sudan; ${ }^{3}$ Unit of Immunology and Microbiology, Soba University Hospital, Khartoum, Sudan; ${ }^{4}$ Unit of Rheumatology, Alribat University Hospital, Khartoum, Sudan; ${ }^{5}$ Unit of Rheumatology, Military Hospital, Omdurman, Sudan

\subsection{6/ard.2010.149096.21}

Rheumatoid arthritis (RA) is characterised by chronic, erosive polyarthritis and by the presence of various autoantibodies. According to our knowledge there are no data published concerning serologic RA phenotypes in Sudan. Our aim in this study is to evaluate the presence of rheumatoid factor (RF) and anticyclic citrullinated peptide antibody (anti-CCP) isotypes in well-characterised cross-sectional hospital-based consecutive cohort of Sudanese RA patients.

Serum samples were collected from 78 RA Sudanese patients (66 female, 12 males) from two rheumatology centers in Khartoum, Sudan between December 2008 and May 2010. Female and male mean age was 48.3 and 55.4, respectively. Median disease duration was 3 years (range 1 month to 58 years). Control groups included 101 Sudanese and 100 Swedish healthy individuals. Diagnosis of this RA cohort done by senior rheumatologists following the 1987 American College of Rheumatology criteria. All patients were on regular follow-up. 69/78 (88.5\%) were treated with diseasemodifying antirheumatic drugs (DMARDs): 21/78 (27\%) with methotrexate, $1 / 78$ (1.3\%) with hydroxychlorokine, $5 / 78(6.4 \%)$ with sulfasalazine and 1/78 (1.3\%) with azathioprine; and $41 / 78(53 \%)$ were treated with different DMARD combinations. 9/78 (11.5\%) were treated with non-steroidal anti-inflammatory drugs only. All autoantibody analyses were performed by ImmunoCap EIA (Phadia AB, Uppsala, Sweden).

Results No differences were found between the Swedish and Sudanese healthy controls for IgG anti-CCP or for for IgM and IgA RF. Among the Sudanese RA patients and their controls, $60.3 \%(47 / 78)$ of Sudanese RA patients and none of the controls were IgG anti-CCP positive ( $p<0.0001$ compared to Sudanese controls). For RF, 31.2\% (24/77) of Sudanese RA patients were IgA RF positive $(p<0.0001)$ and $52.6 \%(41 / 78)$ of them were IgM RF positive $(p<0.0001)$. When we looked for autoantibody combinations, $64.1 \%$ (50/78) has either IgG anti-CCP and/or IgM RF, 62.3\% (48/77) has any of IgG anti-CCP or IgA RF, 57.1\% (44/77) has any of IgM RF or IgA RF and $65.4 \%(51 / 78)$ had any of the three investigated autoantibodies.

There were no statistical difference in erythrocyte sedimentation rate or number of swollen or tender joints between patients with or without IgG anti-CCP, IgM RF or IgA RF, respectively.

Conclusions Our data represent an interim analysis of an ongoing investigation of a larger Sudanese hospital based RA cohort and show IgG anti-CCP positivity in a portion of Sudanese RA patients comparable to results obtained from Swedish RA cohorts. Somewhat fewer RA patients had IgM RF and substantially fewer IgA RF. There were no differences between Swedish and Sudanese control cohorts for any of the investigated autoantibodies. 